Théologiques

Théologiques

\title{
Liturgies africaines et vie
}

\section{François Kabasele Lumbala}

Volume 19, numéro 1, 2011

Théologie africaine et vie

URI : https://id.erudit.org/iderudit/1014185ar

DOI : https://doi.org/10.7202/1014185ar

Aller au sommaire du numéro

\section{Éditeur(s)}

Faculté de théologie et de sciences des religions, Université de Montréal

\section{ISSN}

1188-7109 (imprimé)

1492-1413 (numérique)

Découvrir la revue

\section{Citer cet article}

Kabasele Lumbala, F. (2011). Liturgies africaines et vie. Théologiques, 19(1), 147-162. https://doi.org/10.7202/1014185ar

\section{Résumé de l'article}

En Afrique noire, la vie est le sacré par excellence ; aussi, demeure-t-elle la préoccupation principale de toutes les cérémonies religieuses, déjà dans les traditions, et jusqu'aujourd'hui dans les religions africaines nouvelles : les rituels regorgent d'évocations et de supplications pour la vie ; les lieux de culte sont décorés aux couleurs de la vie (la trilogie « blanc-noir-rouge "); les symboles déployés dans le culte au sein de ces civilisations de l'oralité sont ceux de la fécondité, du triomphe sur la mort, de communion et cohésion sociale : les bananiers, la chaux, les arbres de vie ou arbres aux ancêtres, le feu, etc. Enfin, la manière même de célébrer est des plus vivantes : les assemblées grouillent de monde, la prédication est jalonnée de cris d'acclamation et élaborée avec l'apport de toute l'assemblée, le tambour rythme la prière en réveillant efficacement tous les dormeurs et en mettant debout toute l'assemblée pour la danse qui remet le corps à l'unisson avec l'Esprit et tout le groupe ! Oui, la vie « explose » dans les célébrations africaines. 


\title{
Liturgies africaines et vie
}

\author{
François Kabasele Lumbala" \\ Théologie des religions \\ Institut catholique de la Méditerranée, Marseille (France)
}

Dans un coin de quartier, un vieux morceau de fer, récupéré d'une roue d'automobile qui avait fini ses jours sur un banc de sable, est suspendu à la branche d'un arbre; très tôt le matin, il est vigoureusement battu avec un reste de clé anglaise qui a perdu sa dent, pour appeler à la prière; c'est la cloche de l'Église des Apôtres. À la lueur matinale d'un soleil qui pointe à peine à l'horizon, une ribambelle de gens, hommes et femmes, enfants et adultes, amène son flux vers un hangar tôlé; aussitôt un tambour résonne, des voix s'élèvent dans un chant polyphonique, où se mêlent des cris d'enfants, des imprécations contre le mal, des acclamations en signe d'approbation des paroles du chantre, des battements de mains qui amplifient le rythme; tous chantent; des corps se dandinent tandis que d'autres gesticulent... Nous sommes en Afrique noire. Les nombreux problèmes qui torturent ces peuples n'ont pas eu raison du désir de vivre et d'exprimer la vie, qui transpire de ces assemblées liturgiques.

Mais est-ce le propre de l'Afrique noire? Toute liturgie, dans toutes les religions, est liée à la vie; car le rite, même destiné aux morts, ne fonctionne qu'avec des vivants, et en vue de sauvegarder ou de renforcer la vie individuelle ou sociale. Mais en Afrique noire, ce lien à la vie est encore plus dynamique et s'exprime avec éclat: que ce soit dans la finalité des rites, dans leur déroulement et leur manière d'être organisés, ou encore dans les gestes et les symboles qui y sont mis en œuvre. Ces trois perspectives vont précisément déterminer les trois axes de cette contribution ${ }^{1}$.

* François Kabasele Lumbala est professeur à l’Institut catholique de la Méditerranée (Marseille, France), dans la Section de la théologie des Religions. Il poursuit actuellement ses recherches sur le phénomène religieux et a écrit de nombreux ouvrages et articles sur les cultures d'Afrique noire, ainsi que les rapports entre religions et cultures. Ses publications les plus récentes incluent (2005) Renouer avec ses racines et (2011) Naissances insolites en terre africaine, éditées chez Karthala à Paris.

1. Quand on veut faire comprendre un rite, il ne faut pas faire l'économie de mots et d'images; mais les contraintes de la contribution présente nous ont obligé de résumer 


\section{La vie comme finalité de tous les rites en Afrique}

Nous ne pouvons pas comprendre ce qui se passe dans nos assemblées chrétiennes ou musulmanes actuelles d'Afrique, si nous ne jetons pas un coup d'œil aux traditions religieuses et aux religions d'Afrique noire, en partant de l'Égypte ancienne où la présence et l'influence des Noirs a déjà été attestée (Diop 1954; Obenga 1973; Unesco et Mokhtar 1987). Qu'entend-on par «religion» en Afrique noire? Dans les sociétés d'Occident, quand on parle de "religion ", on songe à un secteur particulier de la vie des hommes, secteur où ils rendent un culte à Dieu et aux Saints. Ce faisant, on distingue d'autres secteurs de la vie, où il ne sera plus question de «culte»; et ce secteur sera alors dénommé "profane», comme n'ayant plus rien à voir avec le culte de Dieu... Ceci est dû à un cheminement particulier de ces peuples, et du concept de «religion". Mais pour nos peuples d'Afrique noire, toute la vie est demeurée «sacrée »; aussi le concept de «religion» en est-il absent. S'il faut parler de «religion », on devra y englober la connaissance et l'explication de l'homme et du monde, la sagesse qui sous-tend et détermine des actions particulières, la morale qui les oriente ou en détermine la valeur. Ainsi en Afrique noire, la «religion» pourrait se définir comme l'harmonisation de toute la vie de l'homme, au rythme de son «alliance» avec Dieu, avec les ancêtres, avec les alliés terrestres, avec la nature, pour la victoire de la vie sur la mort. Ainsi, le signe d'Ankh, symbole de vie, est-il omniprésent dans les manifestations cultuelles et célébrations en Égypte pharaonique. Tout le temps que dura la civilisation égyptienne, l'hiéroglyphe de la croix ansée symbolisa la vie, «ankh" en ancien égyptien. La fameuse hymne d'Akhenaton souligne cette évocation prédominante de la vie dans le culte; voici un petit extrait:

Les êtres de la terre se forment sous ta main comme tu les as voulus.

Tu resplendis, et ils vivent; tu te couches et ils meurent.

Toi, tu as la durée de la vie par toi-même, on vit de toi.

Les yeux sont sur ta beauté jusqu'à ce que tu te couches. (Eggebrecht 2003)

Quatre séries de rites se distinguent dans cette religion: des rites de vénération des ancêtres, des rites d'initiation et des rites d'harmonisation des enfants à titres, des rites de la consécration des saisons ou d'éloigne-

nos descriptions. Voici quelques publications où l'on pourrait trouver leur développement avec plus amples détails et analyses complémentaires: Mulago (1973), Boka Di Mpasi (1980), Lalèyê (1993), Kabasele Lumbala (1996 et 2001), Kabasele Lumbala et al. (2011). 
ment du mal, ainsi que des rites de guérison. Tous ces rites ne sont pas célébrés à un rythme régulier; ils suivent le cours de la vie. C'est une religion "au fil de la vie». L'être humain et sa vie constituent le centre de toutes les préoccupations de ces peuples, centre de la religion et des activités lucratives. C'est ainsi que leurs religions sont qualifiées d' «anthropocentriques ", car ce n'est pas Dieu qui y est recherché, mais la vie de l'homme, une vie pleine.

La vénération des ancêtres se situe aux temps forts de la vie de la communauté, temps forts dictés par une maladie, un événement insolite, la réussite dans une entreprise, la veille d'un long voyage, un rêve où un ancêtre serait venu interpeller les vivants terrestres contre l'oubli, etc. La vénération des ancêtres consiste en l'offrande d'une volaille ou d'un animal de la basse-cour, offrande présidée par l'aîné du groupe, autour duquel tous se rassemblent pour invoquer les ancêtres, pour les remercier, leur demander secours, ou simplement se retremper dans leur mémoire.

Les rites d'initiation consistent à une action d'aide apportée par le groupe à l'individu pour son accomplissement en tant qu'être humain. L'être humain poursuit cet accomplissement jusqu'à la fin de sa vie terrestre; l'initiation lui sert d'instrument dans cette marche qui le conduira d'étape en étape vers la plénitude d'être et son accomplissement. C'est pour cela que l'initiation englobe toute la vie, car la plénitude n'est jamais atteinte sur cette terre, notre vie étant un passage continuel d'un état d'immaturité à un état d'accomplissement. Ces rites se situent à des étapes jugées importantes de la vie de l'être humain: à la naissance, au sevrage de l'enfant, au passage de l'adolescence à l'âge adulte, à l'entrée dans une profession déterminée, à l'intronisation si l'individu accède au rang d'un chef, à l'enterrement et au deuil quand il faudra accompagner l'individu dans le passage vers l'au-delà.

Parmi les rites initiatiques, les plus spectaculaires sont ceux qui se rapportent au regroupement de jeunes adolescents dans un endroit éloigné de la communauté pendant quelques mois; ils y sont soumis à des épreuves; ils y bénéficient des instructions relatives à la vie; et après un rite de mortrésurrection, ils retournent triomphalement à la vie nouvelle. Là où ces regroupements spectaculaires ne se font plus, on a gardé leurs substituts dans les rites d'harmonisation des enfants à titres; ce sont des rites adressés à des naissances «insolites ", hors normes, tels les jumeaux et autres. Ces rites s'échelonnent en effet tous sur trois étapes: entrée en réclusion, période de marge et d'épreuves, retour à la normale. L'entrée en réclusion est marquée par l'habillement spécial où figurent des éléments naturels mis 
en évidence: peaux, kaolin blanc, kaolin rouge, machette rituelle, rasage des cheveux. La mise en œuvre du rite consiste dans l'exécution de chansons et danses qui instruisent sur l'être humain, homme et femme, sur la vie en société, sur Dieu, sur la nature; cette étape de l'instruction est liée aux épreuves, dont l'épreuve finale sera le repas sur la machette, repas présenté par les conductrices de la célébration à la mère des jumeaux; si elle mange de ce qui lui est présenté sur la machette, c'est le signe qu'elle a observé toutes les prescriptions relatives à la croissance de vie des enfants. Cette étape est clôturée par le retour à la normale avec la distribution du repas à toute l'assemblée, ainsi que la série d'imprécations contre le mal, pour que la vie triomphe toujours de la mort.

La consécration des saisons est un rite charnière entre deux saisons qui se succèdent; les femmes sortent de leur maison, légèrement habillées; elles sillonnent les rues de la région, s'arrêtent aux carrefours principaux, et profèrent des imprécations contre le mal. Ces rites sont censés éloigner le mal qui pourrait se glisser dans le rythme de la saison et venir troubler la vie. Ils sont également pratiqués dans les circonstances d'une épidémie qui se serait déclarée dans la région, ou de l'imminence d'une guerre avec des régions voisines.

Enfin, dans la dernière série rituelle, j'ai regroupé les rites de guérison et de communion mystique entre des individus et les Esprits ou divinités. Ces rites sont pratiqués par les prêtresses cilumbu, femmes pour la plupart du temps, qui, durant la transe de la possession par des Esprits des ancêtres, font de la divination et reçoivent des ancêtres des procédés pour guérir telle ou telle maladie... La femme "possédée » par un Esprit reçoit ses malades avec cette consigne: la maladie peut être une conséquence du comportement du patient ou de son entourage; mais elle peut aussi provenir d'une tierce personne: un malfaiteur qui aurait su inoculer le mal dans le corps de sa victime, ou même l'Au-delà qui utiliserait ce signe pour prévenir une catastrophe plus grande. Le décodage se fait dans la célébration, tout comme les procès thérapeutiques.

Dans toutes ces cérémonies traditionnelles, que célèbre-t-on? Autrement dit, qu'est-ce qui requiert commémoraison communautaire et festive? On célèbre tout ce qui concerne l'accroissement de la vie d'un individu ou du groupe: naissance, sortie de maternité ou de l'hôpital, premiers pas d'un enfant, croissance d'un enfant, son passage dans le groupe d'adultes, promotions et intronisations, prémices et premières pluies, nouvelle lune, succès et trophées, mort ou passage dans l'au-delà... La vie est ce qui est recherché partout et en tout. En effet, ces religions placent l'homme au 
centre de toutes les démarches rituelles: il s'agit de l'homme et de la sauvegarde de sa vie; il s'agit moins de louer Dieu que de recourir à Lui pour la sauvegarde de la vie de l'homme qui prie ou des siens pour lesquels il entreprend les démarches.

Si nous revenons aux rituels chrétiens ou musulmans africains d'aujourd'hui, nous constaterons rapidement que les prières africaines s'ingénient à souligner sans cesse le don de la vie; l'évocation de la vie est comme le dénominateur commun de toutes les supplications, la vie étant la valeur suprême. Prenons par exemple le rite "congolais » de la célébration eucharistique, approuvé par Rome depuis 1988. D’entrée de jeu, nous avons l'acte pénitentiel qui implore: «[...] notre vie est diminuée, le mal nous ronge comme une sangsue [...], viens renforcer notre vie »; l'hymne au feu, introduit dans certaines régions à cause du symbole des ancêtres, le déclare ouvertement ${ }^{2}$ : "Venez nous sommes prêts et nous vous prions de vous joindre à nous avec vos forces de vie» (Kabasele Lumbala 1996, 35).

Ailleurs encore, le commentateur souligne: «La célébration eucharistique est si importante que nos Ancêtres doivent y être invoqués, car il y va de l'accroissement de vie en chacun de nous; c'est pourquoi nous nous mettons en communion avec eux» (Kabasele Lumbala 1996, 38).

Chez les Kikuyu, un extrait de leur prière eucharistique mentionne (sur la base d'une prière meru): "Nous quémandons de toi la vie / Un peuple sain, sans maladie / Puisse-t-il avoir des enfants en bonne santé / Donne une vie agréable à nos pères et mères et à nos parents " (Kabasele Lumbala 1996, 53, nous soulignons).

Nous savons combien pour les Africains, vivre c'est donner la vie; aussi demander d'engendrer, c'est demander la vie. Chez les Igbo du Nigéria, un extrait de leur prière eucharistique est éloquent au sujet de la vie, qu'ils citent directement ou suggèrent à travers ses symboles majeurs, tels que la figure ancestrale:

"Créateur du monde, Chi (Dieu), le maître de la vie, nous te louons [...] Notre père, le père de nos ancêtres. La nourriture produite de cette terre, qui nous donne la vie, est ta bénédiction. Que le monde entier fasse l'expérience de la plénitude de vie en toi [...] que nous puissions avoir la plénitude de la vie [...]» (Kabasele Lumbala 1996, 54, nous soulignons).

Ainsi, tant dans la tradition que dans l'actualité des démarches religieuses en Afrique noire, la vie reste soulignée comme objectif principal;

2. On pourra retrouver les citations de différents rituels congolais dans mon ouvrage (Kabasele Lumbala 1996). 
et les Africains devenus chrétiens ne manquent pas de noter et de souligner avec bonheur et satisfaction les paroles de l'Évangile: «je suis venu pour donner la vie, et la vie en abondance» (Jn 3,15-16).

\section{La vie dans le déroulement et l'organisation des rites}

Pour qu'il y ait de la vie dans une célébration, il faut d'abord qu'il y ait du monde; car une célébration où l'on compte à peine une dizaine de membres dans un espace tel que celui d'une église paroissiale donne l'image d'un moribond. Certes chez les guérisseurs et guérisseuses, on ne compte pas des foules, car il s'agit d'une thérapie ciblée, où l'entourage est limité à la famille très proche du patient. Mais même alors, une foule innombrable est censée entourer la prêtresse et le patient: la foule des esprits, non seulement des ancêtres de celui qui est soigné, mais aussi des ancêtres du guérisseur; il y est invoqué également des esprits divers qui peuplent l'univers et qui interviennent dans la procédure de la thérapie.

La plupart des hangars de prière chez diverses Églises de réveil dans nos villes africaines connaissent une ambiance étonnante à cause du nombre de participants: leurs assemblées de prière sont pleines à craquer, et il est fréquent de trouver le surplus des fidèles assis dehors, sur des chaises en plastique, et même sur les trottoirs des rues avoisinantes. Là où il y a du monde, la vie grouille. Et le nombre de «hangars» construits dans les quartiers d'une ville telle que Kinshasa ou Brazzaville est vraiment impressionnant: ils poussent comme des champignons. Un journaliste français en avait compté plus de quatre cents à Brazzaville, il y a une dizaine d'années:

Une génération spontanée d'entrepreneurs en religion a envahi le marché, souvent lucratif, de la prise en charge du malheur, de l'aspiration au changement et de la fabrication du sens [...] ces nouveaux pasteurs et ces «néoprophètes » rodés aux techniques des "télévangélistes » américains officient dans des hangars de tôle, des enclos de palme, des bars reconvertis, des jardins publics ou des domiciles privés [...]. (Soudan 2002, 53)

Qu'y a-t-il dans ce phénomène de la multiplication des lieux de culte et de l'abondance de leur assistance? Pourquoi les Noirs africains achalandent-ils les lieux de culte? Sans doute, la misère y est pour quelque chose! Dans une situation politique et économique désespérante, il est normal que l'homme croyant se tourne d'abord vers l'Au-delà et attende de Dieu un secours que les institutions humaines sont loin de lui garantir. Il suffit d'ouvrir les yeux dans un quartier de ville africaine, comme à 
Kinshasa, Douala, Brazzaville, Abidjan et autres: les paroles bibliques décorent les murs extérieurs des commerces, des pharmacies, des restaurants; des expressions bibliques sont reportées sur les volants des taxis: "l'éternel est mon berger, rien ne saurait me manquer ", "je demeure à l'abri du Très-Haut ", "Dieu soit béni », "Je suis sous la protection du Tout-Puissant». Dans la même perspective, voici un chant catholique, très en vogue, et qui en dit long:

Même si je manque de vêtements, et que je cours dans la nudité, je ne désespérerai jamais, car j'ai à mes côtés, un tailleur de grande renommée, Dieu, mon Dieu. Même si je suis affamé [...], malade, et torturé par les douleurs, je ne désespérerai jamais, car j'ai à mes côtés, un grand médecin, le thérapeute par excellence, Dieu, mon Dieu³ [...].

Mais cela n'explique pas tout le phénomène de l'engouement dans le sacré en Afrique noire! Il y a surtout le fait que la religion en Afrique noire est liée à la vie et qu'il n'y a pas de domaine "sacré» qui soit séparé d'un domaine "profane». C’est toute la vie qui doit s'imprégner du rythme de l'au-delà; car, pour l'Afrique noire, "l'essentiel est invisible pour les yeux», ainsi que le disait Saint-Exupéry. N'est-ce pas pour cela que les célébrations africaines «traînent en longueur" ? C'est le reproche que des chrétiens traditionalistes font aux rites inculturés, leur préférant les célébrations romaines, minutées et chronométrées. En effet, on sait quand commence une célébration africaine, mais on ne peut jamais savoir quand elle se termine!

On prend du temps pour célébrer en Afrique noire, car il y va de la vie et la vie compte plus que toute autre valeur. Un proverbe luba ne dit-il pas: "rien ne peut m'ébranler, tant que je garde la tête sur les épaules" ? On prend du temps pour célébrer en Afrique noire, aussi parce que la vie qui est évoquée dans le rite est incontournable: il faut lui laisser des espaces de jaillissement imprévisible pour nous. D'où de nombreuses improvisations, à cause des événements qui n'avaient pas été prévus lors de la préparation hebdomadaire des liturgies; ces derniers événements doivent trouver de la place dans la prière, même si personne ne s'y était préparée. Ainsi en est-il de l'accueil des étrangers dans la célébration dominicale de nos paroisses: non seulement on les invite à venir devant l'assemblée, pour qu'elle prie sur eux, mais, en outre, on leur donne la parole pour qu'ils disent ce qui les amène et qu'ils puissent témoigner de leur foi dans la trame de leur vie.

3. Rituel luba, ad experimentum (nous traduisons). 
Enfin la manière elle-même de célébrer crie la vie. L'usage du tambour et des xylophones marque énormément les célébrations chrétiennes en Afrique, notamment chez les catholiques, depuis que l'on a permis aux différentes cultures de se déployer durant les célébrations. Le tambour a fait son entrée dans le culte chrétien en Afrique noire depuis plus de cinquante ans. La spécificité du tambour c'est de battre le rythme et de réguler le balancement des corps. Mais bien plus, le tambour est un son qui rend la vie; c'est un son particulier, car ses vibrations sont amplifiées par les petites boules de résine collées sur la peau du tambour. Ce sont ces vibrations qui soulignent la vie comme vibration et mouvement. La résine colle à la main du batteur; mais celui-ci en même temps décolle sa main de la peau du tambour, et la vibration est accentuée davantage. Le son qui s'en dégage est indescriptible, et le moins qu'on puisse en dire est qu'il est d'une attraction irrésistible. Il est encore retravaillé et peaufiné grâce au petit trou laissé sur le bois, à la partie supérieure du tambour; ce petit trou rond est recouvert d'une membrane constituée par un morceau de la peau d'un lézard.

Quand ce tambour est battu par un expert, ou un batteur de talent, il met debout toute l'assemblée. Personne ne peut somnoler dans une salle où ce tambour est battu. Durant des campagnes de guerre traditionnelle, la troupe des combattants apportait toujours un tambour de réveil, qui ranimait les énergies et remontait le moral des combattants. Dans une guerre, la capture du tambourineur augurait l'issue de la bataille. De nombreux mythes et contes ont comme armature principale le tambour, autour duquel la vie est réglée et menée; ainsi le conte Les tambours des guêpes (Sangalayi 1996) que les animaux ont essayé d'arracher. Notons également cette tradition du tambour dont la fabrication avait nécessité le sacrifice d'un humain, lequel y restait enfermé et prisonnier, au service du batteur de ce tambour.

Dans la batterie négro-africaine, au tambour, s'ajoutent presque toujours les grelots, qui n'ont pas de son particulier en rapport avec la mélodie exécutée; leur rôle est de compléter le rythme battu par le tambour. Souvent, c'est le grelot qui commence, pour être suivi par le tambour. Dans une nuit de veillée mortuaire, c'est le grelot qui joue le rôle de réveilleur, surtout quand on atteint les heures difficiles du petit matin.

Seules les Églises protestantes africaines ont résisté à l'adoption du tambour dans leurs célébrations en Afrique noire: pour les missionnaires occidentaux, en effet, le tambour était l'élément quasi principal, le symbole même, du "paganisme ", c'est-à-dire de la culture négro-africaine, telle qu'ils la percevaient alors. Mais cela est en train d'évoluer avec la rencontre des 
différentes communautés africaines au sein du mouvement œcuménique des Églises. L'acharnement des premiers missionnaires contre le tambour des Noirs d'Afrique se comprend par l'énergie vitale, la force d'attraction qui se dégageaient de cet instrument, ainsi que la symbiose qu'il y a entre lui et l'hymnologie traditionnelle où trônaient les Ancêtres et les Esprits.

L'Afrique noire est une des zones de civilisation orale, c'est-à-dire une civilisation où l'esthétique et les procès de la parole trônent comme mode particulier et privilégié de la communication; parole considérée non seulement comme outil, mais surtout comme une puissance de vie. Ainsi la parole est-elle toujours partagée; un proverbe luba dit: «tu peux passer outre un repas pris par un groupe; mais ne passe jamais outre une causerie dans un groupe ». Le prédicateur qui respecte ce style de l'oralité ne tiendra donc pas un monologue, aussi captivant soit-il, à la Bossuet; il s'efforcera toujours de faire intervenir l'assemblée dans son homélie. Concrètement, pendant cette prédication, le peuple intervient par des chants et des répons, après chaque alinéa; souvent, il lancera des bouts de proverbes que le peuple attrape en vol et achève. Ainsi en va-t-il de la parole dans une assemblée liturgique en Afrique noire; il ne s'agira jamais d'un monologue. Aussi est-il rare de voir des gens s'endormir pendant une prédication faite dans le style de l'oralité. L'utilisation des proverbes et des expressions codées que toute l'assemblée reprend dès que le prédicateur les lance, fait du sermon une œuvre collective, et qui se construit au fur et à mesure... L'habileté du prédicateur consistera à conduire l'œuvre là où il avait projeté, tout en y adjoignant l'apport de l'assemblée. Cela donne une vitalité spéciale à nos assemblées de prière en Afrique noire.

Il est souvent reproché à des célébrations africaines de négliger les moments de silence dans la célébration. Effectivement, tout est rempli de "paroles", de musique, de chants, de danses et d'instruments. Il est rare que toute l'assemblée soit en silence; les seuls moments de silence sont ceux de l'écoute à la proclamation de la parole de Dieu et lors des prières sacerdotales. J'ai vu des efforts louables faits par des communautés monastiques à ce sujet: des pauses d'un silence méditatif étaient introduites dans de courtes représentations scéniques où une ou deux moniales exécutaient une danse silencieuse devant toute l'assemblée.

\section{Le déploiement de la vie dans les gestes et symboles}

Les symboles déployés et montrés durant les célébrations en Afrique noire expriment en général le triomphe de la vie sur la mort. Parmi les couleurs, 
c'est surtout la trilogie blanc-rouge-noir; parmi les dessins, nous voyons surtout le cercle, et les rondes; parmi les objets manipulés, nous observons surtout le bananier, le palmier, le balai, la cuillère en bois, la machette ou l'herminette, les calebasses. Cela fait l'effet d'une certaine monotonie dans le décor africain des célébrations; car, en effet, il s'agit toujours du même message: la vie qu'il faut acclamer et accueillir, vie qu'il faut réparer, ou qui demande à être renforcée. La diversité ne viendra que de choses vécues.

Le bananier en particulier est un arbre qui ne renferme aucune ambiguïté: il exprime la continuité et la fidélité de vie, car il reste toujours vert, toute l'année, alors que beaucoup d'arbres perdent leurs feuilles durant la saison sèche. Le bananier exprime également la fécondité, car autour d'un bananier de jeunes pousses sortent pendant que la souche mère s'éteint après avoir donné son fruit. Au Kasayi (Congo), quand un des jumeaux meurt, on l'enterre avec un morceau de tige de bananier à ses côtés, tenant place de son partenaire qui est resté sur terre. Le bananier évoque la vie, quand on voit enterrer le cordon ombilical du nouveau-né au pied d'un bananier: bananier plantain pour l'enfant de sexe masculin, et bananier ordinaire pour l'enfant de sexe féminin (Kabasele Lumbala 1994, 246). C'est toujours le bananier qui évoque la vie, quand les femmes qui procèdent à l'harmonisation des enfants à titres s'en entourent le sein pour la danse.

Aussi, voir un bananier ou une feuille de bananier décorant l'autel ou l'espace de la célébration est un message fort de la vie. Très souvent devant l'autel dans beaucoup de nos églises chrétiennes, lors de grandes fêtes et particulièrement à Noël, on place un grand vase rempli de terre et dans lequel est plantée une jeune pousse de bananier. À défaut d'une jeune plante, il y aura deux grandes feuilles de bananier, déposées dans les deux coins autour de l'autel.

En dehors du bananier, on trouve quelques fois une autre sorte d'arbre tel que le "ficus», symbole de la continuité de la vie; ces arbres sont parfois nommés «arbres de vie», ou «arbres aux ancêtres». Comme le bananier, cet arbre ne perd jamais ses feuilles à la saison sèche. Il figure souvent à proximité des cases rituelles aux ancêtres, qu'on retrouve toujours dans les cours des guérisseurs ou guérisseuses et dans les cours des chefs traditionnels. Outre la vie, ces arbres symbolisent l'harmonie et la bénédiction.

À Tshikapa-Kele (en R. D. Congo), un cabanon aux ancêtres, d'environ $50 \mathrm{~cm}$ de hauteur et $40 \mathrm{~cm}$ de largeur, est construit au pied de l'autel. Ce cabanon est le symbole de la présence des ancêtres à nos côtés. Il manifeste que, dans cet espace, il se joue un drame de vie, un renforcement de 
la vie. Et les ancêtres sont ceux qui, nous ayant transmis la vie, continuent de la soutenir dans l'Au-delà où ils se trouvent auprès de Dieu. Devant le cabanon aux ancêtres, brûle une torche fabriquée avec de la poudre de bois, mélangée à une résine odoriférante (kamonya). La torche est plantée dans du sable que contient une marmite en argile; la torche dans sa marmite est située au milieu des deux fagots de bois placés sur une tablette, devant l'autel. Ce feu est aperçu durant toute la célébration.

Durant la «messe malawienne" à Lilongwe, au monastère des Clarisses, un feu semblable est allumé à côté de l'autel. Une personne est chargée de l'attiser durant toute la célébration. Le feu est un symbole de la présence humaine et de la vie. Il est aussi symbole de la transformation, du renouvellement. La vie est une lutte continuelle, qui demande, pour se maintenir, transformation et renouvellement.

C'est cela qu'évoque le chant exécuté au moment où la torche est allumée par le célébrant principal à Tshikapa-Kele:

Peuple de Dieu, venez, venez, serrons nos coudes, allumons le feu, à ce foyer qui est le nôtre, ce foyer. Oh feu, allume-toi, monte et rayonne [...]. Le feu ne tolère pas l'injustice, cessez donc d'être injustes. Le feu ne tolère pas la jalousie, cessez donc de vous jalouser; [...] nous vous prions de vous joindre à nous avec vos forces de vie. (Kabasele Lumbala, 1996, 35)

Le rappel de la mémoire des ancêtres s'accompagne souvent d'un autre symbole, celui de la chaux; donner la chaux est un geste qui symbolise le souhait de bonne chance dans la vie, la bénédiction de la part des Aînés, la communion avec l'Au-delà. C'est pour cela que tous les chefs traditionnels s'enduisent de chaux lors de leurs sorties; les guérisseurs aussi s'en enduisent pour entamer les rites de guérison. La chaux est ainsi un symbole d'harmonie, de paix et de joie, de la plénitude de la vie, qui se trouve dans la communion avec l'Au-delà.

Outre la chaux, on retrouve sur les murs de beaucoup de nos lieux de culte en Afrique (ceux du moins qui ont les moyens de mettre un artiste à l'œuvre), des symboles bantu de providence et de protection de la vie, tels les triangles, les lances entourant le tabernacle. Sur les vêtements liturgiques, on trouve des «entrelacs» marquant la fécondité, ainsi que des cercles, des spirales, des perles et des cauris, des signes de fécondité et de richesse. Le culte n'est-il pas en effet le lieu où notre vie devient féconde et où nous puisons la véritable richesse de notre être? Ces insignes ont été puisés dans l'ornementation des cours royales traditionnelles: l'ornementation du mobilier utilisé par le chef, l'ornementation portée sur ses armes 
(Neyt 1993; Faik-Nzuji 1993). Dans ces cours royales, ces ornementations conservent jusqu'à présent leur signification, qui est «la protection de la vie».

Le domaine des gestes pendant les célébrations est encore beaucoup plus varié: on y distingue la station debout, la station assise, le regroupement, la procession, l'agenouillement, les mains levées, la danse en général, etc. La station debout est un signe de respect devant l'autorité qui nous aurait convoqués. La station assise est propre à l'écoute, chez les Bantu. La marche en procession est un signe de la vie qui est un passage continu, une marche progressive, sans retour. Ce que confirme un proverbe luba: «la vie n'est pas un marché où l'on va pour en revenir; on y va une fois pour toutes». L'agenouillement symbolise une soumission chez les Bantu; chez certains peuples, on ne parle au chef qu'à genoux; chez d'autres encore, l'épouse ne présente le repas au mari qu'en se mettant à genoux. Tout en luttant contre des attitudes dégradantes par rapport à l'égalité des droits humains et au respect de la dignité de chaque homme et femme, il nous faut cependant remarquer que le respect pour la hiérarchie est une nécessité vitale chez les Bantu et beaucoup d'Africains. Voilà pourquoi la hiérarchie est soulignée dans les rapports sociaux. On le voit par «la peau de léopard» sur l'autel ou sur un mur de l'espace cultuel. La conception du pouvoir en Afrique noire renvoie à l'ordre qu'il faut garder pour que la vie s'épanouisse dans la société. Le léopard est un animal "sacré», symbole du sacré et de la hiérarchie de l'univers; la conception traditionnelle du pouvoir en Afrique noire est au service de la vie, de la croissance de la vie. C'est pourquoi le chef entame sa profession en étant investi sur la peau du léopard; de même les guérisseuses attitrées du dernier degré d'initiation sont investies sur la peau de léopard. Le pouvoir doit être d'abord au service de la vie; c'est pour cela qu'il est, dans les cultures d'Afrique noire, du domaine du «sacré».

Parmi les gestes, la danse occupe une place de choix. La danse dans les cultures occidentales est un élément festif: on danse pour faire la fête, pour s'amuser ou se distraire. En Afrique noire, la danse est un geste particulier, pouvant se retrouver dans une fête, mais aussi en dehors des fêtes, comme dans un deuil, ou durant le travail ou durant le repos; elle est par ellemême un symbole de vie: elle tend à mettre de l'harmonie entre l'homme qui prie et la nature, entre l'homme et le cosmos qui bouge sans cesse; les Africains ont dansé en liturgie chrétienne, car la danse représentait pour eux une manière d'entrer en contact avec l'univers visible et invisible par le canal d'un rythme harmonique; elle est essentiellement mouvement et harmonie, communion de l'esprit et du corps, union du visible et de l'invi- 
sible, et donc communion au rythme imperturbable du monde; la danse négro-africaine est un catalyseur de cohésion dans le groupe. La danse en Afrique noire varie avec l'événement évoqué; au-delà de la chorégraphie et du besoin de faire vibrer le corps au rythme de l'esprit, la danse est un élément d'harmonisation de ce qui se passe au rythme du monde. Pour nos peuples d'Afrique noire, ce monde où nous vivons est considéré comme «bien créé », et donc tournant au rythme divin; il s'agit alors, par la danse, d'imprimer à l'événement, ce rythme de l'univers qui se répercute dans nos corps.

Harmoniser la vie au rythme de Dieu, tel est le rôle principal de la danse négro-africaine durant les célébrations. Aussi danse-t-on de joie (la danse luba du Cyanga), comme on danse de peine (les danses funéraires). On danse l'amour (les danses luba du lutuku) comme on danse la force coercitive, la colère et la haine (les danses exécutées par les chefs ou leurs lieutenants). On danse le repos (danses de repos) comme on danse le travail (chansons de travail). Car partout, il s'agit du rythme et de l'harmonie à rétablir ou à maintenir par ce rythme. Ainsi, les chrétiens africains ont-ils compris que, puisqu'on danse la vie, toute la vie, il était tout à fait normal de danser la vie en Jésus-Christ, qui leur est communiquée dans la liturgie. La prière étant une vie, elle doit être dansée en Afrique.

Non seulement un homme qui danse est un homme vivant, mais la danse qu'il exécute dans une assemblée le met en communion avec les autres; c'est ainsi que, dans un groupe qui danse en Afrique noire, il est difficile de distinguer un boiteux ou un handicapé quelconque; l'unité du rythme est telle que toutes les inégalités sont résorbées dans le mouvement qui se dessine.

Je reste marqué par une scène devant l'hôpital général de Kinshasa: une femme en pleurs, les larmes ruisselant sur son torse nu, les cheveux défaits, un pagne noué autour des reins, dansant autour du brancard qui amenait son fils à la morgue! En pleurant, elle disait: «tu t'en vas, tu me laisses, et tu me connais, moi, faible! Tu t'en vas, tu me laisses, que deviendrais-je sans toi? Amène-moi avec toi, ou bien fais-moi danser, pour que la douleur ne me tue pas!» Elle gesticulait, les mains en l'air, sautant d'un pas à un autre, se dandinant sur ses talons, se frappant de temps en temps la poitrine; ses seins nus amplifiaient le rythme tragique de tout son corps; elle allait et venait d'un bout du brancard à l'autre; sur le brancard gisait le corps sans vie de son fils, recouvert d'un drap blanc.

Ailleurs, il est une coutume de faire circuler le cadavre à travers tous les lieux qu'il avait fréquentés pendant sa vie. Et cela se fait dans une 
longue procession, le corps porté sur un brancard, par des hommes qui, chemin faisant, dansent. Ces démarches rituelles, qui intègrent la danse dans la célébration des funérailles, mettent la vie dans la célébration de la mort. On entend alors en sourdine le poème de Birago Diop (1954): "Non, les morts ne sont pas morts [...] ils sont dans le vent, ils sont dans nos voix qui chantent, ils sont auprès de nos cases $[\ldots]$ ».

\section{Conclusion}

La vie explose dans les célébrations africaines, depuis les traditions profondes du culte aux ancêtres jusqu'aux rites d'initiation, sans oublier les rites de la consécration des saisons et des rites de guérison avec l'intervention des esprits. Les religions nouvelles que la colonisation avait apportées en Afrique noire depuis le $\mathrm{XVI}^{\mathrm{e}}$ siècle n'ont pas réussi à effacer les traditions religieuses de l'Afrique noire; car, même convertis au christianisme, à l'islam ou à des mouvements de réveil hindous et autres, les Noirs africains continuent d'être "animistes", adeptes d'une conception du monde où la vie demeure la valeur principale, le dénominateur commun qui relie tout l'univers, don par excellence du Créateur; aussi la vie de l'homme demeuret-elle la préoccupation principale de toutes les cérémonies religieuses. La manière même de célébrer des Noirs africains reste invariablement liée au mode des civilisations de l'oralité, où la puissance de la parole et du rythme est mise en exergue.

Là où des communautés sont vivantes et ont mis le cap vers l'enracinement de leur foi chrétienne dans les cultures locales, la décoration des lieux de culte, les ornements portés par les membres ou célébrants sont suggestifs de la vie: ainsi les couleurs de la vie, les couleurs symbolisant l'Au-delà et la communion de vie avec le divin, les symboles de fécondité, tels les bananiers, le don total de soi, tels les palmiers, la fidélité et la continuité de la vie, tels les arbres aux ancêtres nommés aussi arbres de vie. Il y a des Églises indépendantes qui n'ont pas adopté l'usage de la construction d'une maison de culte; leur culte se fait au dehors, dans la nature; mais le lieu est bien choisi, pour symboliser que le groupe est le sel de la terre, le levain dans la pâte de la vie; ainsi sont-ils tous habillés de blanc, sous le symbole de la chaux, leur groupe tranchant sur tout l'environnement.

C’est ainsi que l'on retrouve dans toutes les célébrations, en Afrique noire, des séances de guérison, des références continuelles à la vie quotidienne, une affluence remarquable, la danse, le tambour ou les grelots. Il suffit d'assister à un sermon dans une assemblée religieuse africaine pour 
comprendre que personne n'y monopolise la parole, et que cette parole, orchestrée par des cris d'acclamation, par des mélodies, par des instruments, fait de ces assemblées des lieux où la vie éclate, même quand il s'agit d'accompagner le mort à sa dernière demeure.

Mais est-ce l'Afrique qui tient le monopole de l'expression de vie dans les célébrations? La danse n'est-elle pas exécutée dans la chorégraphie rituelle de certains espaces religieux occidentaux et orientaux ? La spécificité de l'Afrique noire, dans tout cela, c'est l'accent mis sur la vie comme préoccupation majeure, comme mode d'expression du sentiment religieux lui-même; l'Afrique noire danse parce que la vie doit être harmonisée avec le rythme de l'univers, rythme de la création elle-même, rythme de Dieu.

\section{Références}

Boka Di Mpasi, L. (1980), "La libération de l'expression corporelle en liturgie africaine ", Concilium, 152/2, p. 77-79.

Diop, C. A. (1954), Nations nègres et cultures, Paris, Présence Africaine.

Eggebrecht, A. (2003), L’Égypte ancienne / trad. par P. Gilbert, Paris, Bordas.

Faik-Nzuji, C. (1993), La puissance du sacré (l'homme, la nature et l'art en Afrique noire), Paris, Maisonneuve et Larose.

Kabasele Lumbala, F. (1994), Alliances avec le Christ en Afrique, Paris, Karthala.

(1996), Liturgies africaines. L'enjeu culturel, ecclésial et théologique, Kinshasa, Facultés catholiques de Kinshasa (Recherches africaines de théologie 14).

(2001), «L'apport des adeptes africains des religions des Ancêtres aux chrétiens d'aujourd'hui », dans Mission de l'Église, Supplément au $\mathrm{n}^{\circ}$ 132, p. 62-66.

Kabasele Lumbala, F. et al. (2011), Naissances insolites en terre africaine (traduit aussi en langue luba), Karthala, Paris.

Laleye, I.-P. (1993), «Les religions d'Afrique noire ", dans J. Delumeau, dir, Le fait religieux, Paris, Fayard.

Mulago, C. M. (1973), La religion traditionnelle des Bantu et leur vision du monde, Kinshasa, Faculté de théologie catholique.

Neyt, F. (1993), Luba, Paris, Dapper.

Овеnga, Т. (1973), L'Afrique dans l'Antiquité. Égypte pharaonique, Afrique noire, Paris, Présence Africaine. 
SANGAlayi (1996), Les tambours des guêpes (Ngoma yà tukwà-matàmbwà), Bruxelles, Sangalayi.

SoudAn, F. (2002), «Le marché florissant du spirituel », dans Jeune Afrique - L'Intelligent, 18-24 novembre.

Unesco et Mokhtar, G. (1987), dir., Histoire générale de l'Afrique. II. Afrique ancienne, Paris, Présence Africaine.

\section{Résumé}

En Afrique noire, la vie est le sacré par excellence; aussi, demeure-t-elle la préoccupation principale de toutes les cérémonies religieuses, déjà dans les traditions, et jusqu'aujourd'hui dans les religions africaines nouvelles: les rituels regorgent d'évocations et de supplications pour la vie; les lieux de culte sont décorés aux couleurs de la vie (la trilogie «blanc-noir-rouge»); les symboles déployés dans le culte au sein de ces civilisations de l'oralité sont ceux de la fécondité, du triomphe sur la mort, de communion et cohésion sociale: les bananiers, la chaux, les arbres de vie ou arbres aux ancêtres, le feu, etc. Enfin, la manière même de célébrer est des plus vivantes: les assemblées grouillent de monde, la prédication est jalonnée de cris d'acclamation et élaborée avec l'apport de toute l'assemblée, le tambour rythme la prière en réveillant efficacement tous les dormeurs et en mettant debout toute l'assemblée pour la danse qui remet le corps à l'unisson avec l'Esprit et tout le groupe! Oui, la vie «explose» dans les célébrations africaines.

\section{Abstract}

In sub-Saharan Africa, life is the Sacred par excellence; hence it remains the main preoccupation of all religious celebrations, both in traditions of old and in today's current new African religions: rituals are crammed with evocations and supplications for life; places of worship are decorated with the colours of life (trilogy "white-black-red"); the symbols displayed during worship in these oral civilizations are those of fecundity, triumph over death, communion and social cohesion: banana trees, white kaolin, trees of life or ancestral trees, fire, etc. Finally, the way of celebrating is one of the liveliest: congregations swarming with people, preaching punctuated with shouts of acclamation and elaborated with participation from the entire congregation, drum rhythms accompanying prayer to awake those who sleep and to throw the entire congregation into dancing, which reunites the whole group as well as the body to the Spirit! Indeed, life "explodes» in African celebrations. 